\title{
Long-term surgical outcomes of liver resection for hepatocellular carcinoma in patients with hepatitis $B$ virus and hepatitis $C$ virus co-infection: A multicenter observational study
}

Hang-Dong JIA, ${ }^{1,2}$, Lei LIANG ${ }^{1,2}$, Chao LI ${ }^{3}$, Han WU ${ }^{3}$, Hong WANG ${ }^{4}$, Ying-Jian LIANG ${ }^{5}$, Ya-Hao ZHOU ${ }^{6}$, Wei-Min GU ${ }^{7}$, Xin-Ping FAN ${ }^{8}$, Wan Yee LAU ${ }^{9}$, Timothy M. PAWLIK ${ }^{10}$, Yong-Kang DIAO ${ }^{1,2}$, Qiu-Ran XU ${ }^{1,2}$, Feng SHEN ${ }^{3}$, Cheng-Wu ZHANG ${ }^{1}$, Dong-Sheng HUANG ${ }^{1,2}$, Tian YANG*1,2,3

'Department of Hepatobiliary Pancreatic and Minimal Invasive Surgery, Zhejiang Provincial People's Hospital (People's Hospital of Hangzhou Medical College), Hangzhou, Zhejiang, China

${ }^{2}$ Departmrnt of Surgery, School of Clinical Medicine, Hangzhou Medical College, Hangzhou, Zhejiang, China ${ }^{3}$ Department of Hepatobiliary Surgery, Eastern Hepatobiliary Surgery Hospital, Second Military Medical University, Shanghai, China ${ }^{4}$ Department of General Surgery, Liuyang People's Hospital, Hunan, China

${ }^{5}$ Department of Hepatobiliary Surgery, The First Affiliated Hospital of Harbin Medical University, Heilongjiang, China

${ }^{6}$ Department of Hepatobiliary Surgery, Pu'er People's Hospital, Pu'er, China

${ }^{7}$ The First Department of General Surgery, The Fourth Hospital of Harbin, Heilongjiang, China

${ }^{8}$ Department of General Surgery, Pingxiang Mining Group General Hospital, Jiangxi, China

${ }^{9}$ Departmrnt of Surgery, Faculty of Medicine, The Chinese University of Hong Kong, Hong Kong, China

${ }^{10}$ Department of Surgery, Ohio State University, Wexner Medical Center, Columbus, OH, USA

Introduction: Hepatocellular carcinoma (HCC) is one of the most serious consequences of chronic hepatitis B virus (HBV) or hepatitis $\mathrm{C}$ virus (HCV) infection. This study sought to investigate long-term outcomes after liver resection for HCC among patients with $\mathrm{HBV} / \mathrm{HCV}$ co-infection (HBV/HCV-HCC) compared with patients with HBV infection (HBV-HCC).

Methods: Patients who underwent curative-intent liver resection for HCC were identified from a multicenter Chinese database. Using propensity score matching (PSM), patients with HBV/HCV-HCC were matched one-to-one to patients with HBV-HCC. Overall survival (OS) and recurrence-free survival (RFS) were compared between the two groups before and after PSM.

Results: Among 2,467 patients identified, 93 (3.8\%) and 2,374 (96.2\%) patients had HBV/HCV-HCC and HBV-HCC, respectively. Compared with patients with HBV-HCC, patients with HBV/HCV-HCC were older, have poorer liver-related characteristics but better tumor-related characteristics. PSM created 88 pairs of patients with comparable liver- and tumor-related characteristics (all $p>0.2$ ). In the PSM cohort, the 3- and 5-year RFS rates in patients with HBV/HCV-HCC were $48.3 \%$ and 38.9\%, which were significantly poorer than patients with HBV-HCC $(61.8 \%$ and $49.2 \%, p=0.037)$. Meanwhile, the 3 - and 5 -year OS rates in patients with HBV/HCV-HCC were also poorer than patients with HBV-HCC (65.4\% and $51.1 \%$ vs. $73.7 \%$ and $63.0 \%)$, with a difference close to be significant between them $(p=0.081)$.

Conclusions: Comparing to patients with HBV-HCC, liver resection resulted in relatively poorer long-term surgical outcomes in patients with HBV/HCV-HCC. 